\title{
Direct Ionization Cross Sections of Uranium Atom By Electron Impact
}

\section{Shivani Gupta ${ }^{1 *} \cdot$ Piyush Sinha ${ }^{1}$}

${ }^{1}$ Department of Physics, H.N.B. Garhwal University, BGR Campus, Pauri, U.K.

*Corresponding Author Email: sgshanu050@gmail.com

Received: 4.10.2021; Revised: 19.10.2021; Accepted: 2.11.2021

CSociety for Himalayan Action Research and Development

\begin{abstract}
A theoretical model for electron impact ionization cross section has been found to be reliable for wide range of atoms is applied in this paper to the Uranium atom. A modified Kim binary encounter Bethe (BEB) method and modified Khare BEB method is employed for calculating electron impact ionization cross sections. The present results so obtained are compared with experimental as well as theoretical results known to the best of our knowledge.
\end{abstract}

Keywords: Electron impact; BEB, Cross sections, Uranium atom.

\section{Introduction}

Uranium atom was discovered by Martin Heinrich Klaproth in 1789. It is a radioactive element with an atomic number 92 and is one of the heavy metals that can be used as a rich source of concentrated energy. The element exists in many rocks in the concentration of 2 to 4 parts per million (ppm) and common in earth's crust as tungsten and tin.

The uranium-235 is essential since it can be split readily and yield lot of energy under certain conditions. Therefore, it is known as fissile and used for nuclear fission. It is mainly used as a fuel in nuclear power reactors for energy generation. The electron induced collision and ionization are the most probable reaction that can take place in the nuclear reactors by using uranium. It is also used by the defence forces to power nuclear submarines and in the manufacture of nuclear weapons.

There are numerous applications of uranium but only few researchers contributed to evaluate its ionization cross sections. The ionization cross section of uranium atom was calculated by taking mean square radii of atomic orbital into consideration (Mann 1967). Firstly, the electron impact ionization cross section of uranium atom has been found experimentally at very low energy range up to $60 \mathrm{eV}$ only (Blackburn and Danielson 1972). Later, the cross section for uranium was evaluated for not more than $500 \mathrm{eV}$ energy (Halle et al 1981). After that no experiment work had been done for ionization cross sections of uranium atom by electron impact.

The ionization cross section of uranium atom and its oxides was also calculated by using spherical complex optical potential formalism 
and then utilizing complex scattering potential ionization contribution method to evaluate total inelastic ionization cross section (Goswami et al 2014). No more results have been reported earlier for electron impact ionization cross section of uranium atom. In this article we are calculating the direct ionization cross sections of uranium atom by utilizing Kim BEB (Kim et al 1994) and Khare BEB (Khare et al 1991, Neelam et al 2010) models respectively. We have modified these methods to make the calculation and evaluation of ionization cross section easier. All the models used in this article has been modified several times (Guerra et al 2012, Kumar 2015) and used to evaluate ionization cross sections of large number of atoms but not that of uranium atom till date.

\section{Theory}

\section{Presently modified Kim BEB model (PMBEB)}

In the present investigation, a different and simpler scaling term is used which requires only one parameter i.e., the binding energy of the particular sub shell for the evaluation of cross sections. A scaling term $1 / \mathrm{T}+\Psi^{\prime}$ (Purohit et al 2021) is adopted where $\Psi^{\prime}$ is a constant that depends on effective nuclear charge $Z^{\prime}$. $Z^{\prime}$ is equal to $\mathrm{Z}-\sigma$ and $\mathrm{Z}$ is the atomic number of atom and $\sigma$ is the screening constant in the particular sub shell. We calculate effective nuclear charge of uranium by applying Slater's Rule. By using this scaling term, we modify the Kim BEB model (Kim et al 1994) and final expression for the non-relativistic direct ionization cross section of atom is now given by (Purohit et al 2021)

$$
\sigma_{P M B E B}=\frac{S}{t+\Psi}\left\{\frac{1}{2}\left(1-\frac{1}{t^{2}}\right) \ln t+\left[\left(1-\frac{1}{t}\right)-\frac{\ln t}{t+1}\right]\right\}
$$

Where, $\mathrm{t}=\mathrm{T} / \mathrm{B}$,

$\Psi^{\prime}=\left(\mathrm{Z}^{\prime} / \mathrm{N}\right) * \mathrm{R}$ and $\Psi=\Psi^{\prime} / \mathrm{B}=\left(\mathrm{Z}^{\prime} /\left(\mathrm{N}^{*} \mathrm{~B}\right)\right) * \mathrm{RandS}=4 \pi \mathrm{a}_{0}{ }^{2} \mathrm{~N}(\mathrm{R} / \mathrm{B})^{2}$

\section{Presently modified Khare BEB model (PMKBEB)}

The ionization cross section of methane was evaluated and the results so obtained were compared with Kim BEB model results (Khare et al 2000). The difference in both the results was about $3 \%$. Khare BEB model has been modified number of times too and a significant contribution was made by giving the nonrelativistic expression for cross sections by electron impact (Tiwariet al 2010). This expression was again subject to modifications in its scaling term (Tiwari et al 2011, Kumar et al 2014). In the present article we have further 
modified the scaling term in Khare BEB model which is simpler than earlier modifications. The scaling term used in Kim BEB and Khare BEB model was same therefore we apply similar modification in Khare BEB model as done in Kim BEB model. The non-relativistic expression for presently modified Khare BEB model (PMKBEB) is

Since, $\sigma_{P M K B E B}=\sigma_{P M B B}+\sigma_{P M M B}+\sigma_{P M t}$

Where $\sigma$ рмвв, $\sigma$ Рммв and $\sigma_{\mathrm{PMt}}$ are the presently modified Bethe, Mott and transversal cross section respectively and the expressions are given by

$$
\begin{aligned}
& \sigma_{P M B B}=\left(\frac{S}{t+\Psi}\right)\left(0.4431\left(1-\frac{1}{t^{2}}\right)-0.5 \ln \left(\frac{1}{t}+\frac{I}{2 m c^{2}}\right)+\frac{1}{2 t^{2}} \ln \left(1+\frac{E}{2 m c^{2}}\right)\right) \\
& \sigma_{P M M B}=\left(\frac{S}{t+\Psi}\right)\left(\left(1-\frac{2}{t+1}+\frac{t-1}{2 t^{2}}\right)+\left(\frac{5-t^{2}}{2(t+1)^{2}}-\frac{1}{t(t+1)}\right)-\left(\frac{(t+1)}{t^{2}} \ln \left(\frac{t+1}{2}\right)\right)\right) \\
& \text { And } \sigma_{P M t}=-\frac{S b_{n l}}{t+\psi}\left(\ln \left(1-\beta^{2}\right)+\beta^{2}\right)
\end{aligned}
$$

Where, $\mathrm{I}$ is the binding energy of the particle. $\mathrm{t}=\mathrm{E} / \mathrm{I}$.

$\Psi=\left(Z^{\prime} /\left(N^{*} B\right)\right)^{*} R$ and $Z^{\prime}$ is the effective nuclear charge, $\beta$ is the ratio of incident velocity $v$ and velocity of light $\mathrm{c}$.

$\mathrm{b}_{\mathrm{nl}}=\alpha p^{-\gamma}$ and $\mathrm{p}=\mathrm{I} / \mathrm{I}_{\mathrm{s}}, \mathrm{I}_{\mathrm{s}}=\mathrm{Z}^{2 * \mathrm{R}}$, Parameters(Khare 2001) $\alpha=0.285 ; \gamma=1.7$.

The binding energy and nuclear charge data used in the present models are listed in Table 1.

Table 1: Binding energy $B$, occupation number $N$, nuclear charge $Z$ ' for uranium atom

\begin{tabular}{|c|c|c|c|c|}
\hline S.No & Binding energy & Sub Shell & $\mathrm{N}$ & $\mathrm{Z}$ \\
\hline 1. & 115611 & $1 \mathrm{~s}_{1 / 2}$ & 2 & 91.65 \\
\hline 2. & 21762 & $2 \mathrm{~s}_{1 / 2}$ & 2 & 89.95 \\
\hline 3. & 20953 & $2 \mathrm{p}_{1 / 2}$ & 2 & 87.85 \\
\hline 4. & 17171 & $2 \mathrm{p}_{3 / 2}$ & 4 & 87.85 \\
\hline 5. & 5553 & $3 \mathrm{~s}_{1 / 2}$ & 2 & 82.85 \\
\hline 6. & 5187 & $3 \mathrm{p}_{1 / 2}$ & 2 & 80.75 \\
\hline 7. & 4308 & $3 \mathrm{p}_{3 / 2}$ & 4 & 80.75 \\
\hline 8. & 3733 & $3 \mathrm{~d}_{3 / 2}$ & 4 & 70.85 \\
\hline 9. & 3557 & $3 \mathrm{~d}_{5 / 2}$ & 6 & 70.85 \\
\hline 10. & 1446 & $4 \mathrm{~s}_{1 / 2}$ & 2 & 66.35 \\
\hline 11. & 1278 & $4 \mathrm{p}_{1 / 2}$ & 2 & 64.25 \\
\hline 12. & 1050 & $4 \mathrm{p}_{3 / 2}$ & 4 & 64.25 \\
\hline 13. & 785 & $4 \mathrm{~d}_{3 / 2}$ & 4 & 52.85 \\
\hline 14. & 743 & $4 \mathrm{~d}_{5 / 2}$ & 6 & 52.85 \\
\hline 15. & 396 & $4 \mathrm{f}_{5 / 2}$ & 6 & 47.95 \\
\hline 16. & 386 & $4 \mathrm{f}_{7 / 2}$ & 8 & 47.95 \\
\hline 17. & 329 & $5 \mathrm{~s}_{1 / 2}$ & 2 & 36.45 \\
\hline 18. & 261 & $5 \mathrm{p}_{1 / 2}$ & 2 & 34.35 \\
\hline 19. & 203 & $5 \mathrm{p}_{3 / 2}$ & 4 & 34.35 \\
\hline
\end{tabular}




\begin{tabular}{|c|c|c|c|c|}
\hline 20. & 110 & $5 \mathrm{~d}_{3 / 2}$ & 4 & 20.85 \\
\hline 21. & 101 & $5 \mathrm{~d}_{5 / 2}$ & 6 & 20.85 \\
\hline 22. & 6 & $5 \mathrm{f}_{3 / 2,5 / 2}$ & 3 & 19.8 \\
\hline 23. & 52 & $6 \mathrm{~s}_{1 / 2}$ & 2 & 13.8 \\
\hline 24. & 34 & $6 \mathrm{p}_{1 / 2}$ & 2 & 11.7 \\
\hline 25. & 24 & $6 \mathrm{p}_{3 / 2}$ & 4 & 11.7 \\
\hline 26. & 6.1 & $6 \mathrm{~d}_{3 / 2}$ & 1 & 3 \\
\hline 27. & 6 & $7 \mathrm{~s}_{1 / 2}$ & 2 & 3 \\
\hline
\end{tabular}

In the present work, we have made calculations for the ionization cross sections from ionization threshold to $5000 \mathrm{eV}$ for each sub shell by using modified Kim BEB and modified Khare BEB modelswhich are summed up to yield the cross sections for that particular energy. The screening constant used in these models has been obtained semi empirically. In the next section, we will show our results obtained by using these models and compare it with the available experimental as well as other theoretical results.

\section{Results and Discussion}

In the present investigation the direct ionization cross sections have been calculated for the uranium atom by the modified Kim BEB and modified Khare BEB models for incident energy varying from threshold ionization energy to about $5000 \mathrm{eV}$. The binding energies used to calculate cross sections is listed in Table 1.

Figure 1 shows the comparison of present crosssections for uranium along with the experimental data given by Blackburn and Danielson(1972) and of Halle et al(1981) as well as theoretical results of Mann et al(1967) and Goswami et al(2014).
It is evident from the graph that the theoretical results obtained by Mann et al(1967)agree well with the present data and peaks obtained by Blackburn and Danielson(1972)experimentally are also comparable with the peak valuescalculated by present model. While the present cross sectionsoverestimate the data of Halle et al(1981).At higher energy the present results are in good agreement with Goswami et $\mathrm{al}(2014)$.The results of both the present approaches are in good agreement with each other as well. In PMKBEB model the cross sectionsarelarger than those in PMBEB model at low energies whilebeyond $100 \mathrm{eV}$ these align.

\section{Conclusion}

The proposed models are the modifications in Kim BEB and Khare BEB models for the electron impact ionization of atoms. The direct ionization cross sections of uranium atom up to $5000 \mathrm{eV}$ are calculated and are comparable with the available experimental and theoretical data. We can conclude that slight modification in the scaling term has considerably improved the agreement with experimental and other theoretical results. Moreover,as the present model depends only on one parameter that is 
binding energy hence there is less scope of error. From the plots it can be seen that the results of modified Kim BEB and modified Khare BEB models merge at high energies. The present approach can be extended to more atoms, ions and molecules to yield reliable data.

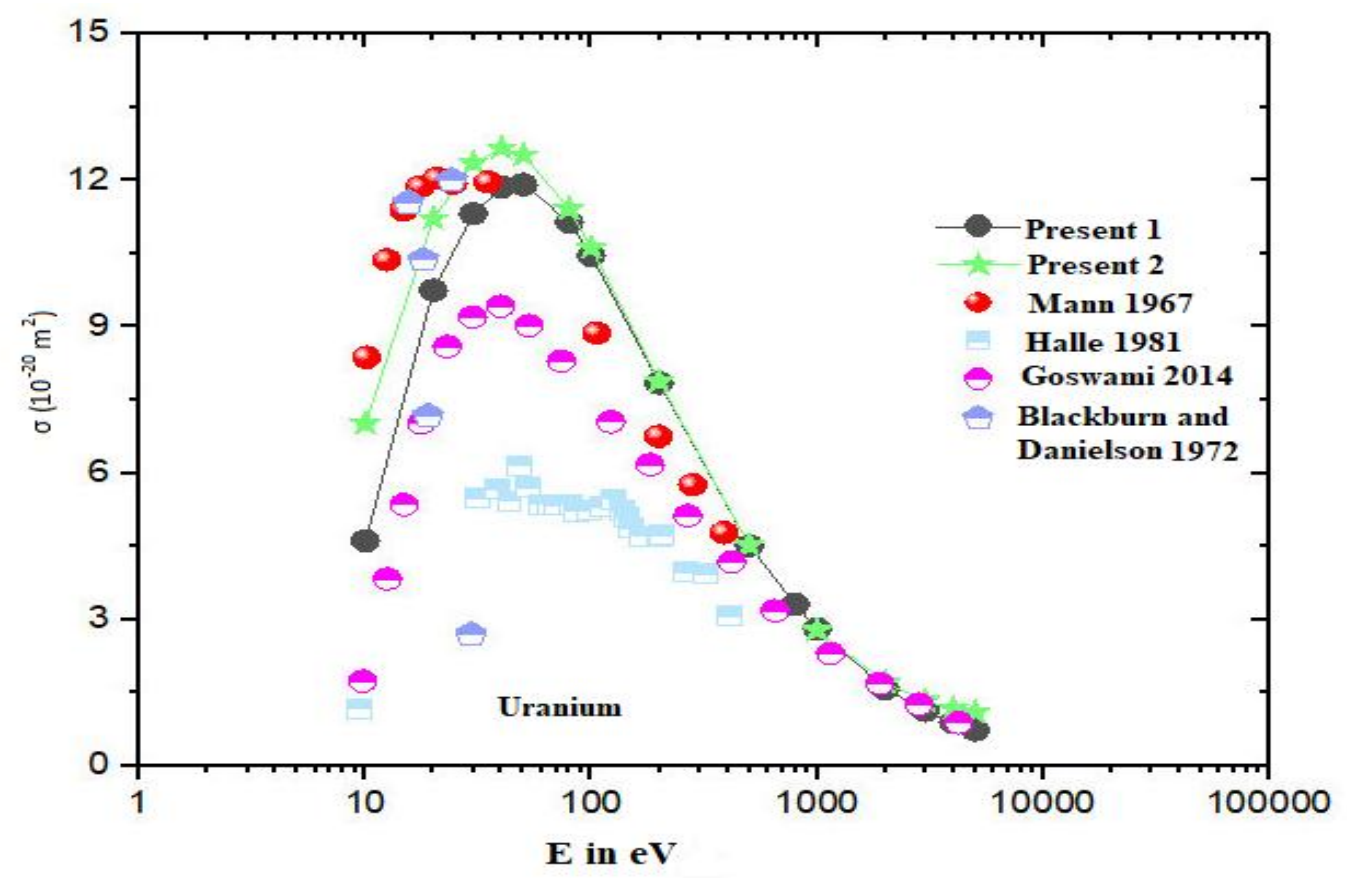

Figure 1 Present 1 ( - ) is presently modified Kim BEB(PMBEB) and present $2(-)$ represents presently modified Khare BEB (PMKBEB) results respectively.

\section{Acknowledgement}

One of the authors SG is grateful to acknowledge the financial support of this research by the Department of Science and Technology (DST), New Delhi through research Grant No. C/3257/IFD/2017-18.

\section{References}

Blackburn P E and Danielson P M (1972)

Electron impact Relative Ionization Cross Sections and Fragmentation of $U, U O$,
UO2 and UO3 Pp. 12; The Journal of Chemical Physics, 56: 6156.

Goswami B, Naghma R and Antony B (2014) Calculations of electron impact total ionization cross sections for tungsten, uranium and their oxide radicals; International journal of Mass Spectroscopy, 372: 8-12.

Guerra M, Parente F, Indelicato P and Santos J P (2012) Modified binary encounter Bethe model for electron-impact ionization; 
International journal of Mass Spectroscopy, 7: 3131 .

Halle J C, Lo H H and Fite W L (1981) Ionization of uranium atoms by electron impact Pp. 4; Physical Review A, 23: 1708.

Mann J B (1967) Ionization cross section of the elements Calculated from Mean Square Radii of Atomic Orbitals Pp. 5; The Journal of Chemical Physics, 46: 1646.

Khare S P, Sharma M K and Tomar S (1999) Electron impact ionization of methane; Journal Physics B: Atomic Molecular Optical Physics, 32: 3147-3156.

Khare S P, Tomar S and Sharma M K (2000) Electron impact molecular ionization; Journal Physics B: Atomic Molecular Optical Physics, 33: L59-L61.

Khare S P, (2002) Introduction to the theory of collisions of electrons with atoms and molecules; (Kluwer Academic Press, New York).

Kim Y K and Rudd M E (1994) Binaryencounter-dipole model for electron-impact ionization Pp. 5; Physical Review A, 50: 3954.

Kim Y K, Santos J P and Parente F (2000) Extension of the binary-encounter-dipole model to relativistic incident electrons; Physical Review A, 62: 052710.
Kumar Y and Kumar M (2014) Inner-shell ionization cross section of gold by electron and positron impact; Journal At Molecular Science, 5: 352-360.

Kumar M (2015) K- shell L-shell and M- shell ionization cross section of bismuth atom by electron impact; International Journal of Advanced Research in Engineering and technology (IJARET), 6: 34-39.

Purohit G, Kato G, Murakami I, Gupta S and Sinha P (2021) Calculation of electron induced ionization cross sections of fusion plasma relevant material: $W$ atoms; The European Physical Journal D, 75: 9.

Tiwari N, Kumar Y and Tomar S (2010) Electron impact ionization cross sections of $\mathrm{C} 2 \mathrm{H} 2$ and $\mathrm{CH}$ molecules at low and high energy range; Journal At Molecular Science, 1: 301-307.

Tiwari N and Tomar S (2011) K-shell ionization cross sections of light atoms due to electron impact; Journal At Molecular Science, 2: 109-116. 